\title{
Transplantation in the single ventricle population
}

\author{
Louise A. Kenny ${ }^{1}$, Fabrizio DeRita ${ }^{1}$, Mohamed Nassar ${ }^{1,2}$, John Dark ${ }^{1,3}$, Louise Coats ${ }^{1,4}$, Asif Hasan ${ }^{1}$ \\ ${ }^{1}$ Adult Congenital and Paediatric Heart Unit, Freeman Hospital, Newcastle upon Tyne NHS Foundation Trust, Newcastle Upon Tyne, UK; \\ ${ }^{2}$ Cardiothoracic Surgery Department, Alexandria University, Alexandria, Egypt; ${ }^{3}$ Institute of Cellular Medicine, Faculty of Medical Sciences, \\ ${ }^{4}$ Cardiovascular Research Centre, Institute of Genetic Medicine, Newcastle University, Newcastle upon Tyne, UK \\ Correspondence to: Louise A. Kenny. Adult Congenital and Paediatric Heart Unit, Freeman Hospital, Newcastle upon Tyne NHS Foundation Trust, \\ Freeman Road, Newcastle Upon Tyne NE7 7DN, UK. Email: LKenny@doctors.net.uk.
}

\begin{abstract}
The single ventricle patient population comprises the most complex cohort presenting to the cardiac transplant team, in terms of demographics, anatomic substrate, and unique physiology. It is also the most rapidly growing diagnostic group presenting for heart transplantation. In this manuscript, we aim to describe the changing landscape of transplantation in single ventricle conditions through reflection on our own institution's practice and experience, alongside contemporary literature review. Single ventricle patients are heterogeneous in terms of age, anatomic diagnosis and physiology according to surgical stage of repair. Progress in surgical palliative strategies has impacted upon the present composition of the population, with growing numbers of hypoplastic left heart syndrome patients and those with late physiology failure following Fontan completion. Multiple prior surgeries, immunological sensitivity and multi-organ involvement impart high peri-operative risk but can be mitigated in part by careful pre-operative planning by a dedicated multidisciplinary team addressing issues such as planning of concurrent reconstructive surgery, minimizing the post-operative effect of collaterals, timely harvesting, oversizing of donor organs to minimize graft failure, and strategies to address anticipated post-operative elevation in pulmonary vascular resistance. Determining optimal timing for transplant in these patients remains unclear, but understanding the risk of alternative surgical options can help guide decision making with regards to listing.
\end{abstract}

Keywords: Single ventricle (SV); Fontan; heart transplant; congenital heart disease (CHD)

Submitted Jan 15, 2018. Accepted for publication Jan 21, 2018.

doi: 10.21037 /acs.2018.01.16

View this article at: http://dx.doi.org/10.21037/acs.2018.01.16

\section{Historical perspective}

In 1967, just 3 days after the world's first heart transplant was performed by Christian Barnard, the first infant heart transplant was undertaken by Adrian Kantrowitz in New York (1). The child with Ebstein malformation died just a few hours post-operatively; however, this pioneering operation began the journey of heart transplantation in the management of patients with single ventricle (SV) conditions. Almost 3 decades later, whilst William Norwood and his team in Boston were navigating the technical and ethical complexities of palliating the SV heart, Leonard Bailey enraged animal rights activists in California by implanting a baboon heart into a neonate with hypoplastic left heart syndrome (HLHS) (2). The story of baby Fae, who lived for 21 days, remains a popular case in medical ethics and whilst the publicised outcry halted the xenotransplant programme, it incited Bailey's team to lead the way for primary orthotopic heart transplant (OHT) in SV neonates.

The role of transplantation in the management of SV conditions remains unclear. Jack Rychik in his seminal paper "Forty years of Fontan Operation: A Failed Strategy" does not even mention OHT in long-term management (3). This may reflect a general perception that heart transplantation is an unattractive option for these patients. Yet, rather than dismissing it, we should instead ask the question of where transplantation best fits in the management strategy for SV conditions. 

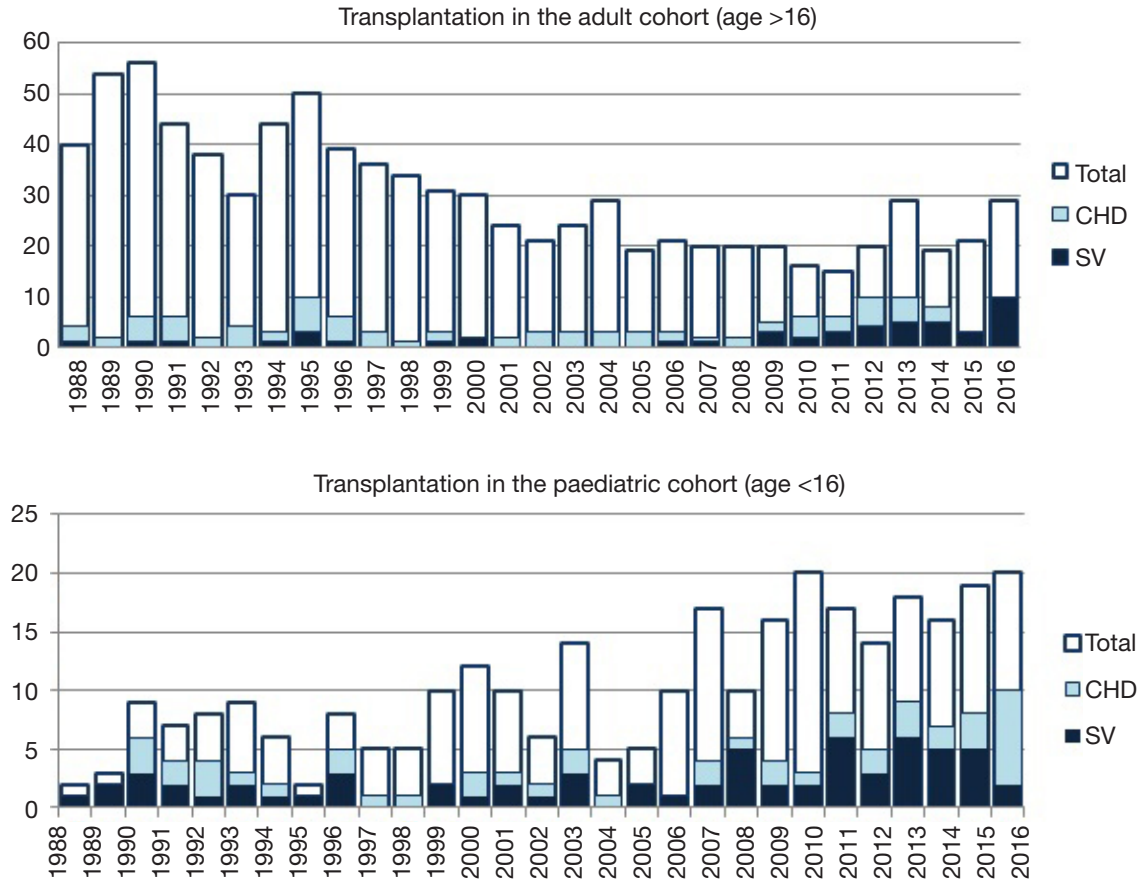

Figure 1 Freeman Hospital orthotopic heart transplants from 1999 to 2016 for adults and children, demonstrating the increasing numbers of SV patients. SV, single ventricle; CHD, congenital heart disease.

\section{Introduction}

As univentricular infants increasingly survive early staging surgery and Fontan completion, thus reaching adolescence and adulthood, the demographic of this complex patient group is shifting, with a demonstrable effect upon the population presenting for OHT. Whilst transplant-free survival following Fontan surgery exceeds $80 \%$ at 20 years, eventual failure of the $\mathrm{SV}$ is for most inevitable and thus prompts referral for $\mathrm{OHT}$, regarded as the 4th stage in management $(4,5)$. The SV patient, however, at all ages and stages of palliation poses a cerebral and technical challenge to the transplant team due to heterogeneous anatomy and surgical history, high immunological exposure, and unique physiology which often persists through the peri-operative period.

The purpose of this manuscript is to depict the changing landscape of transplantation in SV conditions, reflecting upon our own institution's practice and experience and reviewing the lessons we have learned along the way.

\section{The changing profile of the SV transplant population}

Congenital heart disease (CHD) is a growing indication for OHT in the adult population, with a $40 \%$ increase over the past 15 years (ISHLT) (6). The proportion of OHTs performed for SV pathology is currently unclear, but it is predicted that this heterogeneous group of patients will account for $70-80 \%$ of CHD transplantation in the future (7).

In our institution, our first transplant for SV physiology was performed in 1988 in an adult with double inlet left ventricle, who remains alive and well nearly 3 decades later. Since then, our program of adult and paediatric transplantation in univentricular conditions has grown, with the emerging adult group gaining greater prominence in recent years (Figure 1). In our first 15 years, we performed 34 SV OHTs, comprising 35\% of CHD transplants (8). In the subsequent 14 years, this doubled to $75 \mathrm{SV}$ OHT, comprising over $50 \%$ of CHD transplants, and included one heart-liver transplant (9).

\section{The heterogeneity of the SV population}

The SV population is a diverse group comprising multiple etiologies, cardiac architectures, and stages of surgical reconstruction. The term "univentricular" is used to describe any construct unable to support independent 
pulmonary and systemic circulations, despite the fact that in the vast majority of cases two ventricles exist, albeit one of rudimentary size (10). Conditions included, and thus presenting for OHT, are single atrioventricular connection, common atrioventricular connection with unbalanced ventricular mass, double inlet or double outlet ventricle, and single outlet with intact ventricular septum. Functionally univentricular situations also occur as a result of etiologies such as Ebstein anomaly.

The spectrum of conditions presenting for OHT has changed over recent decades, primarily due to successful palliation of HLHS, with the failing morphological right ventricle now overtaking the morphological left ventricle as the predominant substrate (11). By 1981, on publication of the first successful Norwood palliation, the primary indication for paediatric OHT was unoperated HLHS $(12,13)$, an indication that has become increasingly uncommon due to the remarkable reduction in peri-operative mortality from Norwood procedures. Consequently, it is now failing physiology following Fontan completion that is the predominant indication for OHT in children and adults with CHD (14).

SV heart failure necessitating OHT presents across a wide range of ages, at various stages of surgical repair, and in various manifestations, from salvage to elective. Evolving surgical solutions to address the morphological substrate have further compounded the heterogeneity of the population by adding varying surgical architecture, including pulmonary artery bands; ductal stents; Blalock Taussig, Sano and Glenn shunts, hemi-Fontans, atriopulmonary, lateral tunnel and total caval connections, and more. This degree of complexity requires the transplant surgeon and team to remain flexible, innovative and always questioning when managing this challenging patient group.

\section{Pertinent issues in SV heart transplantation}

\section{Technical considerations}

While palliation has revolutionised management and outlook for SV patients, it is often the anatomical and physiological consequences of staging procedures, not initial morphological diagnosis, that challenges the transplant surgeon most.

The SV patient of the modern era has, in most institutions, undergone at least one prior cardiac surgery. Re-entry of the thorax becomes increasingly challenging and hazardous with each prior sternotomy and is associated with a 4-fold elevation of in-hospital mortality (15). Adhesions, destabilising bleeding on resternotomy, and difficult cannulation may be encountered. Collaterals and arteriovenous malformations complicate bleeding and flows on cardiopulmonary bypass. Detailed pre-operative knowledge of the cardiac and extra-cardiac anatomy is critical as frequently vascular reconstruction is needed and generous donor tissue must be available with the explanted donor graft to facilitate this. Anomalies that may require specific reconstructive approaches include abnormal situs, heterotaxy, persistent left superior vena cava, and anomalous systemic or pulmonary venous drainage. Longer operative, cardiopulmonary bypass, and ischaemic times should be anticipated.

\section{Listing}

Patients with CHD wait longer on the list than non-CHD patients and carry a higher waiting list mortality (16). This, combined with limited high-risk mechanical support options to bridge to OHT and unrelenting progression of physiological failure, should prompt early consideration of transplantation in apparently stable patients (17). Markers used to assess progression of ventricular failure in acquired heart failure are not predictive in SV physiology, nor do they indicate the degree of pulmonary vasculature abnormalities, cyanosis, collaterals, liver and kidney impairment, protein losing enteropathy (PLE), plastic bronchitis $(\mathrm{PB})$ or other systemic consequences. In selected patients, the risks and benefits of improving physiology through high-risk upstaging, down-staging or Fontan conversion must be balanced against the potential risks and benefits of OHT.

\section{Unique complexities of SV patients}

\section{HLHS}

Despite notable advances in HLHS palliation, $30 \%$ of those with morphological right ventricles still die in their first year and transplant free survival for the HLHS patient is only $64 \%$ at 3 years of age $(18,19)$. But poor organ availability and combined high waiting list and early post OHT mortality continues to drive palliation as the primary management approach in most centres, except in those with severe ventricular dysfunction or valve regurgitation (20). Despite this, HLHS is still reported as the most common diagnosis for infants undergoing OHT for CHD in the 
current era (21). With predicted expansion of this cohort, it is important to recognize that these patients present younger and with a shortened interval since palliation, reflecting the poor durability of the systemic right ventricle.

\section{The Fontan patient}

With progressive improvement in survival following early palliation, we have seen a 5 -fold increase in Fontan patients, with a projected $60 \%$ increase over the next decade (22). This expanding group poses a distinct challenge when presenting late due to numerous multisystem consequences (23).

Patients presenting for OHT can be divided into two groups: those with impaired ventricular function, and those with preserved ventricular function with failed Fontan physiology (FFP). In our adult patients undergoing transplant for FFP, we identified preserved ventricular function as an independent predictor of mortality (24). Griffiths et al. verified this in a younger cohort when they reported 1-year survival after OHT in impaired ventricular function patients at $88.9 \%$, compared to $56.2 \%$ in those with preserved ventricular function, $80 \%$ of whom had PLE or PB (25).

\section{PLE and PB}

PLE and PB present exclusively in patients with preserved ventricular function following Fontan completion (25). PLE is characterized by abnormal serum protein loss into the gut lumen. Failing integrity of the enteral mucosa in PLE, which is yet to be fully understood, leads to reduced vascular oncotic pressure resulting in ascites and pleural effusions, abnormalities in calcium metabolism secondary to hypoalbuminemia, loss of clotting factors deranging the coagulation cascade, and lymphopenia which may predispose to serious infections triggered by post-OHT immunosuppression. These patients, typically adolescents or adults, present late with insidious decline, often "tolerating" their symptomatology. PLE without transplantation carries a $50 \%$ mortality at 5 years; resolution of PLE postOHT is reported in $100 \%$ of early survivors, highlighting its development as a compelling indication for transplant $(26,27)$. However, destabilization of multiple homeostatic mechanisms leads to high peri-operative risk which must be considered when counselling patients (4,28). Finding better ways to monitor progression in this apparently "stable" group will in the future better inform timing of OHT, which has led some to suggest that OHT is not indicated in this cohort (29).

Patients with $\mathrm{PB}$, typically children, present acutely with airway obstruction from acellular casts secondary to leakage of protein through breached bronchial mucosa. This life-threatening complication of FFP is rare and as such is poorly understood, but is reported in small series to increase peri-transplant mortality (30).

\section{Liver disease}

Fontan associated liver disease, resulting from chronically elevated venous pressure and sub-optimal cardiac output in response to stressors, manifests variably as fibrosis, cirrhosis, focal nodular hyperplasia, hypervascular lesions, and hepatocellular carcinoma. Portal hypertension, varices and ascites may result, but surprisingly synthetic dysfunction is often late.

In our institution, all Fontan patients considered for OHT are discussed at a multi-disciplinary meeting attended by a hepatologist and in selected cases, a hepatobiliary surgeon alongside the OHT team. To date, this has resulted in us performing one heart-liver transplant for this indication (9). Our practice is to consider patients with cirrhosis but normal synthetic function, normal hepatic venous anatomy, good liver volume and no significant portal hypertension or hepatocellular carcinoma for OHT alone (31).

\section{Pulmonary vascular resistance (PVR)}

The detriment to PVR of non-pulsatile flow is 2-fold: first, loss of pulsatile distal recruitment, and second, reduced endothelial nitric oxide release. Further complications arise from arteriovenous malformations secondary to loss of hepatic factor. Arteriovenous malformations causing severe cyanosis are reviewed for consideration of coiling prior to OHT, as persistence post-transplant can lead to high cardiac output primary graft failure (32).

Evaluation of PVR in the Fontan patient is hampered by the unequal and low flow state in the pulmonary vasculature. Microvascular occlusion is common, as are arteriovenous malformations, leading to erroneous trans-pulmonary gradients. Although difficult to evaluate, elevated PVR is a key mechanism in FFP and unmasking can occur postOHT following restoration of pulsatile flow with detrimental effects on right ventricular function $(33,34)$. Early studies demonstrated higher post-transplant mortality with elevated 


Box 1 Risk factors for orthotopic heart transplant in single ventricle
patients
Common requirement for pulmonary artery reconstruction
Multiple previous operations (sternotomies and thoracotomies)
High anti-human leukocyte antibody titres
Renal and hepatic dysfunction
Elevated pulmonary vascular resistance unmasked post
operatively
Failing Fontan physiology including
Protein losing enteropathy (and consequences for homeostasis)
Plastic bronchitis
Refractory ascites

Heterotaxy (unquantified infection risk posed by polysplenia or asplenia)

PVR; more recent evaluation is less convincing, suggesting better appreciation of perioperative management strategies for PVR and right ventricular dysfunction (35). While guidelines recommend exclusion of patients with transpulmonary gradients greater than $15 \mathrm{mmHg}$, it is crucial to account for reversibility of elevated PVR with pulmonary vasodilators which may permit heart-alone transplant in higher pulmonary pressures situations (36).

\section{Mortality in SV heart transplantation}

SV physiology in adults has been shown to carry a 3-fold higher risk of in-hospital mortality following OHT compared with biventricular pathologies (16), prompting discussion of appropriate allocation of limited donor resources $(21,29,37)$. Early complications, relating to transplant complexity, contribute substantially to the mortality of both adults and children and have been reported across the literature $(24,28,38)$. Survivors have comparable outcomes to all CHD recipients.

It is perhaps not surprising that the greatest burden of mortality occurs in the immediate post-OHT period, given the combination of highly complex re-operative surgery and the often poor, pre-operative status of the patient. Early deaths are typically multifactorial due to the complex substrate. Multiple previous operations predispose to dangerous sternal re-entry. Necessity for adhesion dissection and great vessel reconstruction leads to longer cardio-pulmonary bypass times and organ ischaemic times, contributing to graft failure (39). Long-standing venous congestion leads to hepatic dysfunction, renal compromise, and pathologic protein losses, which in combination with iatrogenic immunosuppression increases susceptibility to infections. Previous immunological sensitization, secondary to high usage of blood and homograft products and reflected in elevated panel reactive antibodies, has been associated with a higher risk of rejection and graft failure (40). Additionally, elevated pulmonary vascular resistance may not be apparent until post-transplant, compromising the donor right ventricle and leading to early graft failure (41).

Concerning outcomes have been reported for the Fontan group with an 8.6 relative risk increase of early mortality post-transplant in adult and paediatric patients (28). However, recent interrogation of the Paediatric Heart Transplant Study Database showed improved 1-year survival from $71 \%$ [1993-2006] to 89\% [2007-2014] for those undergoing OHT for FFP, reaching comparable outcomes to other CHD patients (42). Other paediatric registry data has shown comparable outcomes between Fontan and nonFontan SV OHT patients $(27,43,44)$. In our own adult data of OHT for FFP, we have shown a 2-fold improvement in 30 -day survival from $40 \%$ to $76 \%$ over similar eras (24). A 2017 meta-analysis, comprised of 351 patients (age range, 6.6-23.8 years) with Fontan circulations revealed 1- and 5 -year survival of $80 \%$ and $71 \%$, whilst ISHLT data reports 1 - and 5 -year survival post OHT for all-cause CHD in adults at $77 \%$ and $67 \%(6,45)$.

While some sub-groups of Fontan patients demonstrate higher mortality, outcomes approaching non-Fontan CHD survival are now being seen, particularly in the younger population. Patients with heterotaxy have poor long-term outcomes post-transplant and OHT should be considered judiciously in this group (46). Other recognized but unquantified risk factors are shown in Box 1 .

\section{Optimal timing of heart transplant in SV patients}

The optimal positioning of OHT in the management strategy of the failing SV circulation is yet to be determined, with conflicting evidence from small cohorts clouding the issue. In a recent review, HLHS infants with previous palliation were shown to have poorer outcomes compared to those with unoperated HLHS (21). Infants undergoing OHT for failed first stage 1 at less than 1 month of age were at highest risk. This identifies HLHS as a sub-group in whom early failure could be anticipated, and primary OHT pursued. 
OHT as a rescue for early failure at less than 6 months following Fontan completion has been associated with higher mortality than OHT after Glenn shunt (27). Surgeons with growing experience, may push the boundaries of who and when to up-stage, resulting in a higher number of early Fontan failures being referred for transplant. In previous eras, these patients with immediate compromise who are usually extremely sick (47), were not considered for transplant and therefore underwent Fontan take-down. Mortality of OHT in this scenario is reported to be as high as $44-66 \%(29,48)$. In our institution, OHT proved to be viable although technically challenging, in a limited cohort in this situation (49). Our experience with this challenging group has influenced our practice of deliberately oversizing donor hearts to compensate for high PVR, and judicious interpretation of pulmonary vascular investigations as a predictive measure of early failure. Better defining the patient at high risk of acute failure after Fontan completion could avoid the prohibitively high in-hospital mortality associated with Fontan take-down (50) and rescue OHT if semi-elective OHT were offered instead of Fontan completion.

Early and late survival is reported to be optimal when OHT is performed following Glenn shunt but before Fontan completion rather than at shunt stage, or even worse, Fontan stage (29). Following the Glenn shunt, the detrimental effect of shunt physiology including ventricular overload and coronary steal have been reduced and the multi-organ consequences of Fontan completion which contribute to early post-operative mortality are avoided (24). Higher pulmonary artery pressures, heterotaxy and atrioventricular valve regurgitation should alert the assessing team to potential early failure and prompt consideration of elective bridge to OHT from Glenn. The timing of OHT in the late failing Fontan remains highly controversial and an area of developing expertise.

\section{Lessons}

* In light of the limited donor pool and high waiting list mortality, an understanding of indications and outcomes for SV patients undergoing OHT is essential to appropriately position OHT in the management pathway of this disparate group;

* Improvement in our own and others peri-operative management of these patients parallels the evolution of the multidisciplinary team with a dedicated interest in this group (including surgeons, transplant physicians, intensivists, adult and paediatric cardiologists, immunologists, hepatologists, specialist nurses and psychologists) in combination with development of specific regular forums for discussion and ongoing review of these patients to address modifiable risk factors and implement plans for the non-modifiable using a systems based approach;

* In surgical practice, we have developed techniques required for complex re-entry to the chest whilst concurrently ensuring timely harvesting of the donor organ to minimise ischemic times. We have employed peri-operative strategies for anticipated high PVR including up-sizing donor weight (8). Supporting this challenging physiology through the transition from SV circulation to the transplanted biventricular state is an ongoing learning process.

\section{Conclusions}

OHT in the SV patient group is a rapidly developing field with growing need for dedicated expertise in allied specialties. The heterogeneous and changing patient demographic requires a personalised, systems-based approach with innovative surgical and medical solutions to achieve optimal outcomes. Understanding of the timing and role of OHT in the lifetime management of this group is developing with the acknowledgement that it is a limited resource required by a very challenging and high-risk cohort of patients.

\section{Acknowledgements}

The authors would like to thank Gwen Taylor, Dr. G Parry and Dr. AJ Hanson.

\section{Footnote}

Conflicts of Interest: The authors have no conflicts of interest to declare.

\section{References}

1. Kantrowitz A, Haller JD, Joos H, et al. Transplantation of the heart in an infant and an adult. Am J Cardiol 1968;22:782-90.

2. Bailey LL, Nehlsen-Cannarella SL, Concepcion W, et al. Baboon-to-Human Cardiac Xenotransplantation in a Neonate. JAMA 1985;254:3321-9. 
3. Rychik J. Forty years of the fontan operation: A failed strategy. Semin Thorac Cardiovasc Surg Pediatr Card Surg Annu 2010;13:96-100.

4. Khairy P, Fernandes SM, Mayer JE, et al. Long-term survival, modes of death, and predictors of mortality in patients with Fontan surgery. Circulation 2008;117:85-92.

5. Gamba A, Merlo M, Fiocchi R, et al. Heart transplantation in patients with previous Fontan operations. J Thorac Cardiovasc Surg 2004;127:555-62.

6. Lund LH, Edwards LB, Kucheryavaya AY, et al. The registry of the International Society for Heart and Lung Transplantation: thirty-first official adult heart transplant report--2014; focus theme: retransplantation. J Heart Lung Transplant 2014;33:996-1008.

7. Jayakumar KA, Addonizio LJ, Kichuk-Chrisant MR, et al. Cardiac transplantation after the Fontan or Glenn procedure. J Am Coll Cardiol 2004;44:2065-72.

8. Irving C, Parry G, O'Sullivan J, et al. Cardiac transplantation in adults with congenital heart disease. Heart 2010;96:1217-22.

9. Duong P, Coats L, O'Sullivan J, et al. Combined heartliver transplantation for failing Fontan circulation in a late survivor with single-ventricle physiology. ESC Heart Failure 2017;4:675-8.

10. Frescura C, Thiene G. The New Concept of Univentricular Heart. Front Pediatr 2014;2:62.

11. Backer CL, Russell HM, Pahl E, et al. Heart transplantation for the failing Fontan. Ann Thorac Surg 2013;96:1413-9.

12. Norwood WI, Lang P, Casteneda AR, et al. Experience with operations for hypoplastic left heart syndrome. J Thorac Cardiovasc Surg 1981;82:511-9.

13. Boucek MM, Mathis CM, Razzouk A, et al. Indications and contraindications for heart transplantation in infancy. J Heart Lung Transplant 1993;12:S154-8.

14. Stout KK, Broberg CS, Book WM, et al. Chronic Heart Failure in Congenital Heart Disease. Circulation 2016;133:770-801.

15. Gandhi R, Almond C, Singh TP, et al. Factors associated with in-hospital mortality in infants undergoing heart transplantation in the United States. J Thorac Cardiovasc Surg 2011;141:531-6.

16. Karamlou T, Hirsch J, Welke K, et al. A United Network for Organ Sharing analysis of heart transplantation in adults with congenital heart disease: outcomes and factors associated with mortality and retransplantation. J Thorac Cardiovasc Surg 2010;140:161-8.

17. Everitt MD, Donaldson AE, Stehlik J, et al. Would access to device therapies improve transplant outcomes for adults with congenital heart disease? Analysis of the United Network for Organ Sharing (UNOS). J Heart Lung Transplant 2011;30:395-401.

18. Daly KP. Finding the correct role for heart transplant in the treatment of hypoplastic left heart syndrome. J Heart Lung Transplant 2016;35:299-300.

19. Newburger JW, Sleeper LA, Frommelt PC, et al. Transplantation-Free Survival and Interventions at 3 Years in the Single Ventricle Reconstruction Trial. Circulation 2014;129:2013-20.

20. Chrisant MR, Naftel DC, Drummond-Webb J, et al. Fate of infants with hypoplastic left heart syndrome listed for cardiac transplantation: A multicenter study. J Heart Lung Transplant 2005;24:576-82.

21. Everitt MD, Boyle GJ, Schechtman KB, et al. Early survival after heart transplant in young infants is lowest after failed single-ventricle palliation: a multi-institutional study. J Heart Lung Transplant 2012;31:509-16.

22. Coats L, O'Connor S, Wren C, et al. The single-ventricle patient population: A current and future concern a population-based study in the North of England. Heart 2014;100:1348-53.

23. De Rita F, Crossland D, Griselli M, et al. Management of the Failing Fontan. Semin Thorac Cardiovasc Surg Pediatr Card Surg Annu 2015;18:2-6.

24. Murtuza B, Hermuzi A, Crossland DS, et al. Impact of mode of failure and end-organ dysfunction on the survival of adult Fontan patients undergoing cardiac transplantation. Eur J Cardiothorac Surg 2017;51:135-41.

25. Griffiths ER, Kaza AK, Wyler von Ballmoos MC, et al. Evaluating Failing Fontans for Heart Transplantation: Predictors of Death. Ann Thorac Surg 2009;88:558-63; discussion 563-4.

26. Silvilairat S, Cabalka AK, Cetta F, et al. Protein-losing enteropathy after the fontan operation: Associations and predictors of clinical outcome. Congenit Heart Dis 2008;3:262-8.

27. Bernstein D, Naftel D, Chin C, et al. Outcome of listing for cardiac transplantation for failed Fontan: A multiinstitutional study. Circulation 2006;114:273-80.

28. Lamour JM, Kanter KR, Naftel DC, et al. The Effect of Age, Diagnosis, and Previous Surgery in Children and Adults Undergoing Heart Transplantation for Congenital Heart Disease. J Am Coll Cardiol 2009;54:160-5.

29. Michielon G, Parisi F, Squitieri C, et al. Orthotopic heart transplantation for congenital heart disease: an alternative for high-risk fontan candidates? Circulation 2003;108 
Suppl 1:II140-9.

30. Gossett JG, Almond CS, Kirk R, et al. Outcomes of cardiac transplantation in single-ventricle patients with plastic bronchitis: A multicenter study. J Am Coll Cardiol 2013;61:985-6.

31. Greenway SC, Crossland DS, Hudson M, et al. Fontan-associated liver disease: Implications for heart transplantation. J Heart Lung Transplant 2016;35:26-33.

32. Krishnan US, Lamour JM, Hsu DT, et al. Management of aortopulmonary collaterals in children following cardiac transplantation for complex congenital heart disease. J Heart Lung Transplant 2004;23:564-9.

33. Egbe AC, Connolly HM, Miranda WR, et al. Hemodynamics of Fontan Failure. Circ Heart Fail 2017;10:e004515.

34. Mitchell MB, Campbell DN, Ivy D, et al. Evidence of pulmonary vascular disease after heart transplantation for Fontan circulation failure. J Thorac Cardiovasc Surg 2004;128:693-702.

35. Maxwell BG, Sheikh AY, Ajuba-Iwuji CC, et al. Pulmonary vascular resistance index and mortality after paediatric heart transplant. Cardiol Young 2015;25:1141-7.

36. Gazit AZ, Canter CE. Impact of pulmonary vascular resistances in heart transplantation for congenital heart disease. Curr Cardiol Rev 2011;7:59-66.

37. Kulkarni A, Neugebauer R, Lo Y, et al. Outcomes and risk factors for listing for heart transplantation after the Norwood procedure: An analysis of the Single Ventricle Reconstruction Trial. J Heart Lung Transplant 2016;35:306-11.

38. Davies RR, Russo MJ, Yang J, et al. Listing and Transplanting Adults With Congenital Heart Disease. Circulation 2011;123:759-67.

39. Iyengar AJ, Sharma VJ, Weintraub RG, et al. Surgical strategies to facilitate heart transplantation in children after failed univentricular palliations: The role of advanced intraoperative surgical preparation. Eur J Cardiothorac Surg 2014;46:480-5.

40. Auerbach SR, Smith JK, Gralla J, et al. Graft survival is

Cite this article as: Kenny LA, DeRita F, Nassar M, Dark J, Coats L, Hasan A. Transplantation in the single ventricle population. Ann Cardiothorac Surg 2018;7(1):152-159. doi: 10.21037/acs.2018.01.16 better without prior surgery in cardiac transplantation for functionally univentricular hearts. J Heart Lung Transplant 2012;31:987-95.

41. Michielon G, Carotti A, Pongiglione G, et al. Orthotopic Heart Transplantation in Patients with Univentricular Physiology. Curr Cardiol Rev 2011;7:85-91.

42. Simpson KE, Pruitt E, Kirklin JK, et al. Fontan Patient Survival After Pediatric Heart Transplantation Has Improved in the Current Era. Ann Thorac Surg 2017;103:1315-20.

43. Kanter KR, Mahle WT, Vincent RN, et al. Heart Transplantation in Children With a Fontan Procedure. Ann Thorac Surg 2011;91:823-9; discussion 829-30.

44. Kovach JR, Naftel DC, Pearce FB, et al. Comparison of risk factors and outcomes for pediatric patients listed for heart transplantation after bidirectional Glenn and after Fontan: An analysis from the Pediatric Heart Transplant Study. J Heart Lung Transplant 2012;31:133-9.

45. Tabarsi N, Guan M, Simmonds J, et al. Meta-Analysis of the Effectiveness of Heart Transplantation in Patients With a Failing Fontan. Am J Cardiol 2017;119:1269-74.

46. Larsen RL, Eguchi JH, Mulla NF, et al. Usefulness of cardiac transplantation in children with visceral heterotaxy (asplenic and polysplenic syndromes and single rightsided spleen with levocardia) and comparison of results with cardiac transplantation in children with dilated cardiomyopathy. Am J Cardiol 2002;89:1275-9.

47. Backer CL. Rescuing the failing Fontan. Heart 2016;102:1077-8.

48. Petko M, Myung RJ, Wernovsky G, et al. Surgical reinterventions following the Fontan procedure. Eur J Cardiothorac Surg 2003;24:255-9.

49. Chaudhari M, Sturman J, O’Sullivan J, et al. Rescue cardiac transplantation for early failure of the Fontantype circulation in children. J Thorac Cardiovasc Surg 2005;129:416-22.

50. Knott-Craig CJ, Danielson GK, Schaff H V, et al. The modified fontan operation. J Thorac Cardiovasc Surg Jun;109:1237-43. 